\title{
Refinamiento de la punta nasal y base alar con la utilización de punch e injertos. Técnica Personal
}

\author{
Refinement of the nasal tip and alar base with the use \\ of punch and grafts
}

José Durán

\begin{abstract}
RESUMEN
En el siguiente trabajo se realiza la presentación de una técnica de refinamiento de la punta nasal y base alar utilizando punch y debilitando la crura alar con la extracción de injertos individuales que luego se colocan en los domos.

Este refinamiento estaría indicado, sobre todo, en pacientes que presentan cruras externas prominentes en las que se pretende realizar un debilitamiento o refinamiento de la misma utilizando punch de 4 o $5 \mathrm{~mm}$, a la vez se utilizan los cartílagos extraidos como injertos en los domos, con la intención de mejorar los puntos luminosos de los mismos. La misma se realiza como complemento de una rinomodelación simple o combinada (resección de giba ósea y cartilaginosa)
\end{abstract}

Palabras claves: punta nasal ancha, base alar ancha, injertos punch en los domos.

REVISTA ARGENTINA DE CIRUGÍA PLÁSTICA 2018;24(3):121-125. DOI/10.32825/RACP/201803/0121-0125

\section{INTRODUCCIÓN}

Existen varias técnicas de refinamiento de la crura externa con injertos de cartílago en la punta que incluyen técnicas de rinoplastia cerrada y abierta.

En esta publicación se presenta esta técnica como complemento de la rinomodelación con hilo simple o combinada. Indicada en pacientes que presentan la punta nasal ancha a expensas de la crura externa. Una buena alternativa de refinamiento de la misma utilizando en este caso un punch a la vez que se utilizan los injertos cilíndricos obtenidos, para realizar injertos individuales en los domos pretendiendo mejorar los puntos luminosos de los mismos.

En cuanto al estrechamiento de la base alar también podemos utilizar los punch para extraer tejido dermograso, cilíndricos y simétricos sin involucrar la mucosa nasal.

\section{INDICACIONES}

Rinomodelaciones simples y combinadas con punta nasal ancha en pacientes con piel delgada o intermedia y base alar ancha.

1. Cirujano plástico. Presidente de la Regional del Noroeste (NOA), SACPER.

$\triangle$ Correspondencia: Dr. José Durán. Coronel Puch 497 San Salvador de Jujuy - Jujuy CP 4600. joseduran_cirugia@hotmail.com

\section{MATERIAL Y MÉTODO}

Punch de 3, 4 o $5 \mathrm{~mm}$.

PDS 4-0.

Se utilizó anestesia local con Xilocaína al 2\% con epinefrina.

Esta técnica se realiza desde junio de 2018. Se intervino hasta la fecha a un total de dieciséis pacientes compuestos por diez refinamientos de punta nasal con rinomodelación combinada con hilo. En los seis restantes se realizó refinamiento de base alar, cuatro de ellos como complemento de rinomodelación y dos de forma aislada.

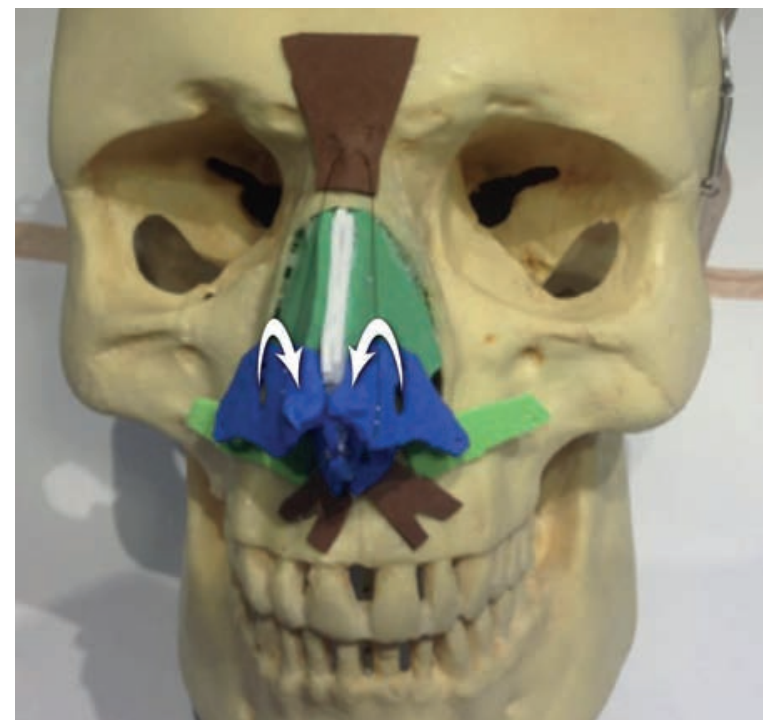

Figura 1. Maqueta explicativa. 

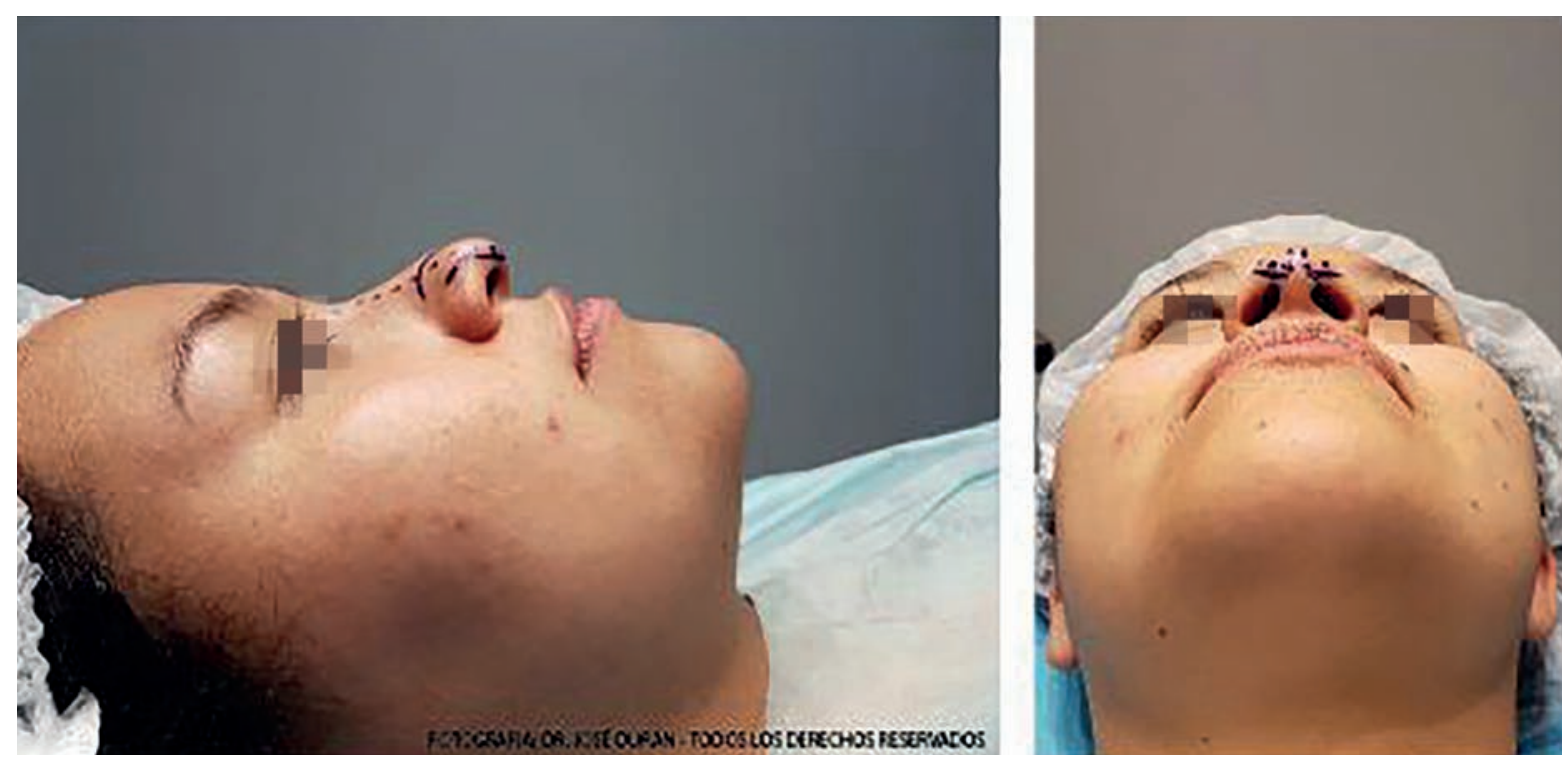

Figura 2. Preoperatorio.
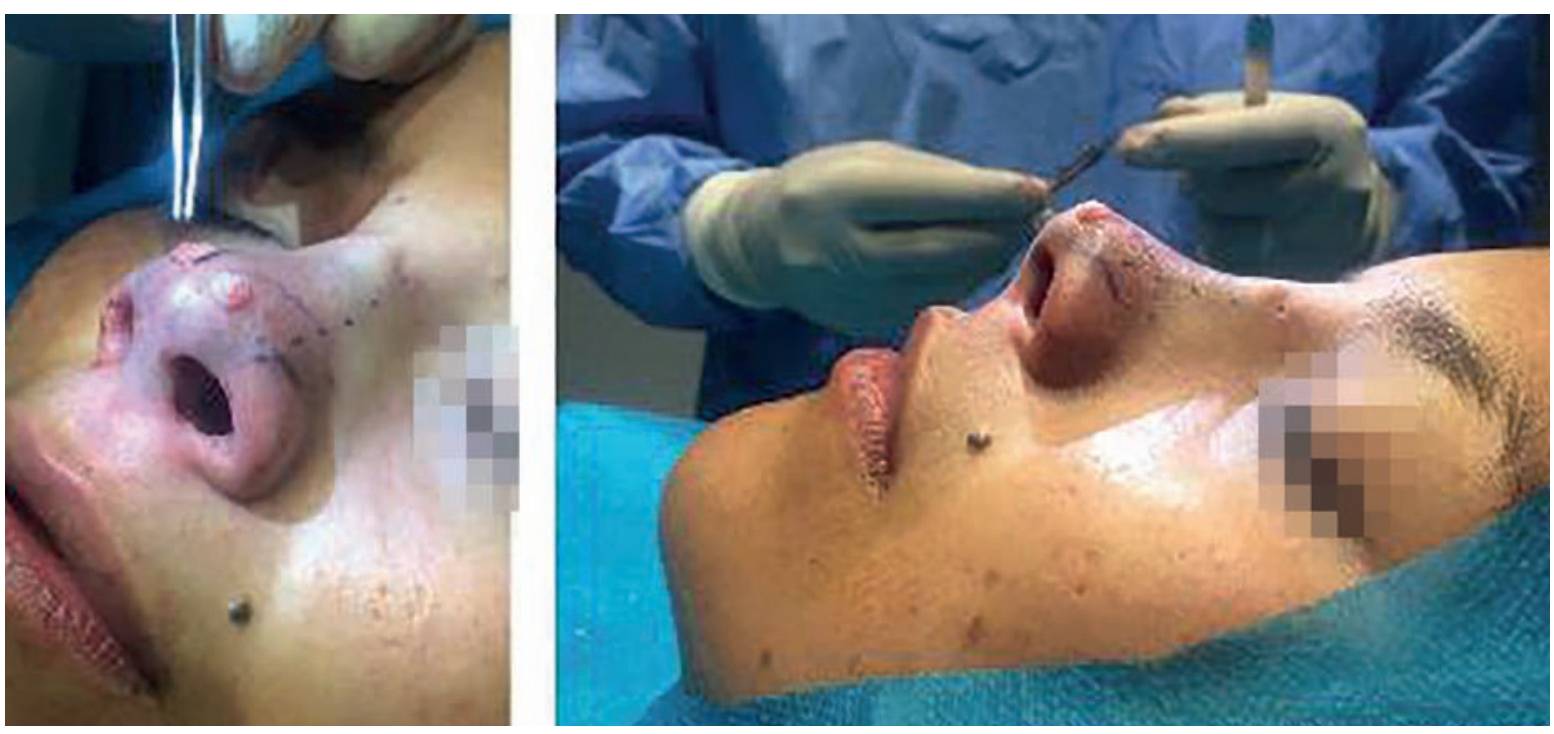

Figura 3. Presentación de injertos.
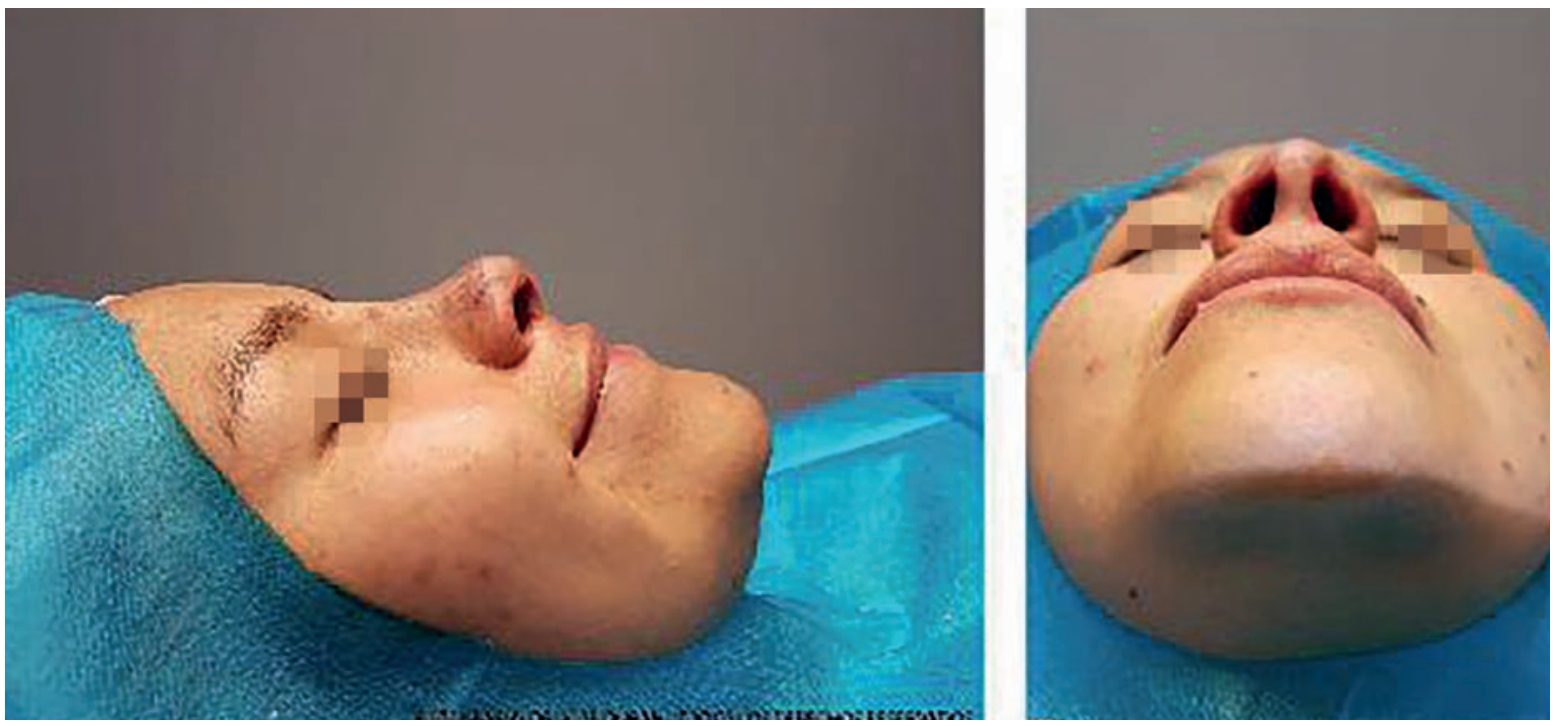

Figura 4. Posoperatorio inmediato. (Ver video de procedimiento en: https://youtu.be/PKS2UxaPWR8) 

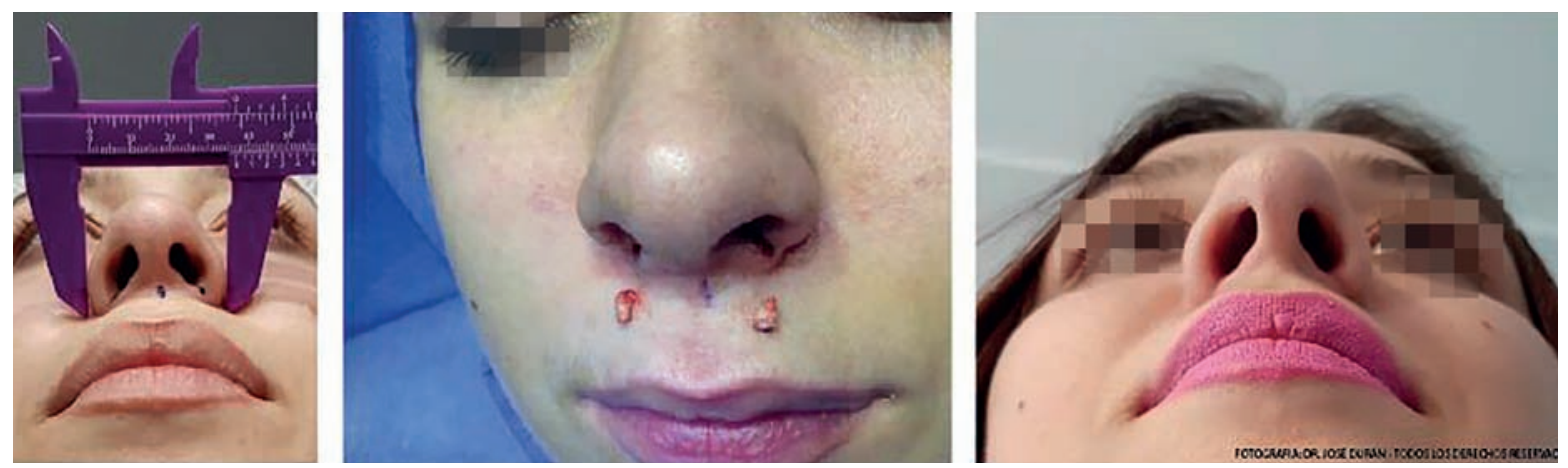

Figura 5. Base alar ancha. Punch de 3 mm. A. Preoperatorio. B. Posoperatorio inmediato. C. Posoperatorio 5 días.
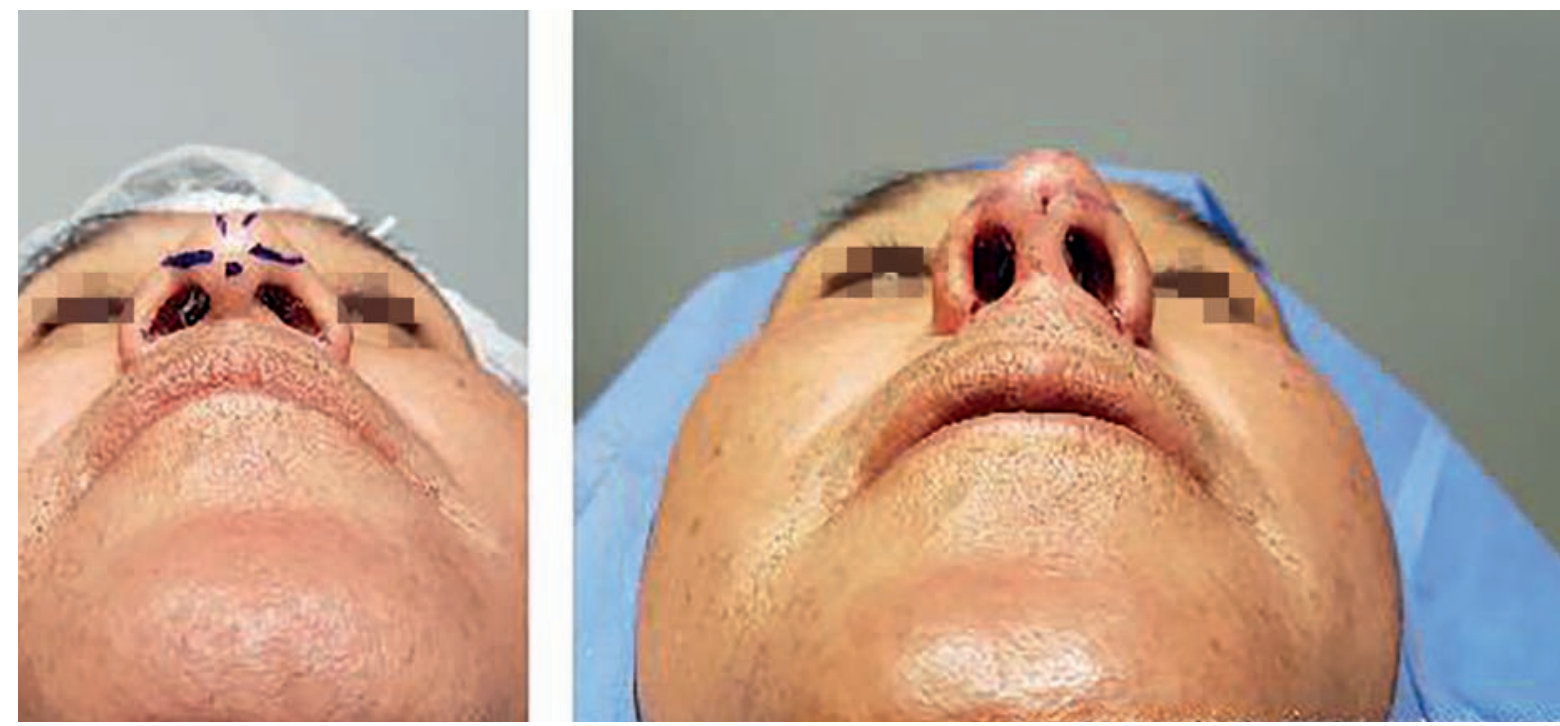

Figura 6. Base alar ancha. Punch $4 \mathrm{~mm}$. A. Preoperatorio. B. Posoperatorio inmediato.
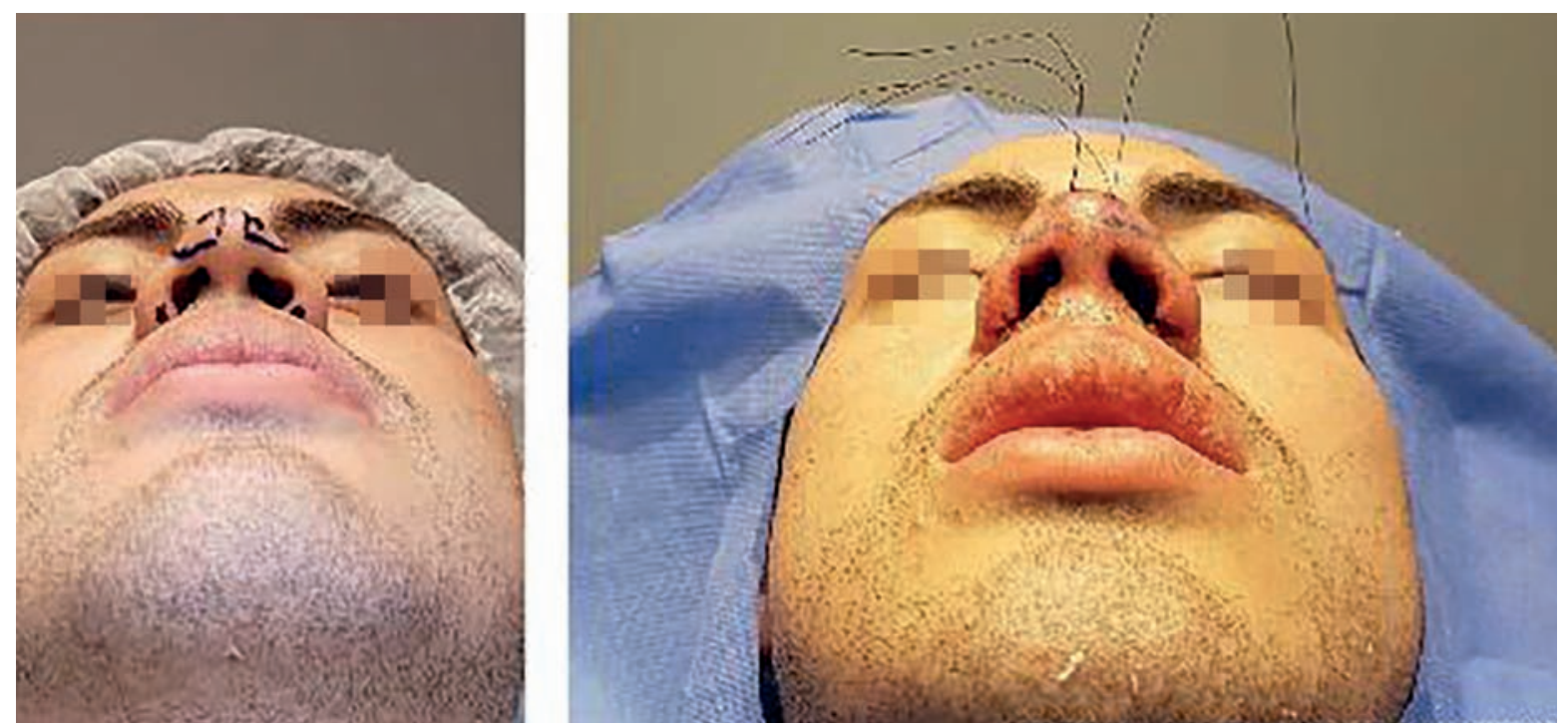

Figura 7. Base alar ancha. Punch $5 \mathrm{~mm}$. A. Preoperatorio. B. Posoperatorio inmediato.

\section{TÉCNICA QUIRÚRGICA}

Se utiliza en todos los casos anestesia local con xilocaina al $2 \%$ con epinefrina. Se realiza marcación previa de la crura externa de la zona que se pretende debilitar, siempre como complemento de una rinomodelación.
En estos casos se agrega infiltración de la mucosa nasal. Se realiza una incisión mínima con hoja $\mathrm{N}^{\circ} 15$, intracartilaginosa, luego se procede a la disección con tijera curva de iris, a continuación se reseca el cartílago de la crura externa, en el área previamente marcada, con la utilización de punch de 4 o $5 \mathrm{~mm}$ dependiendo del 


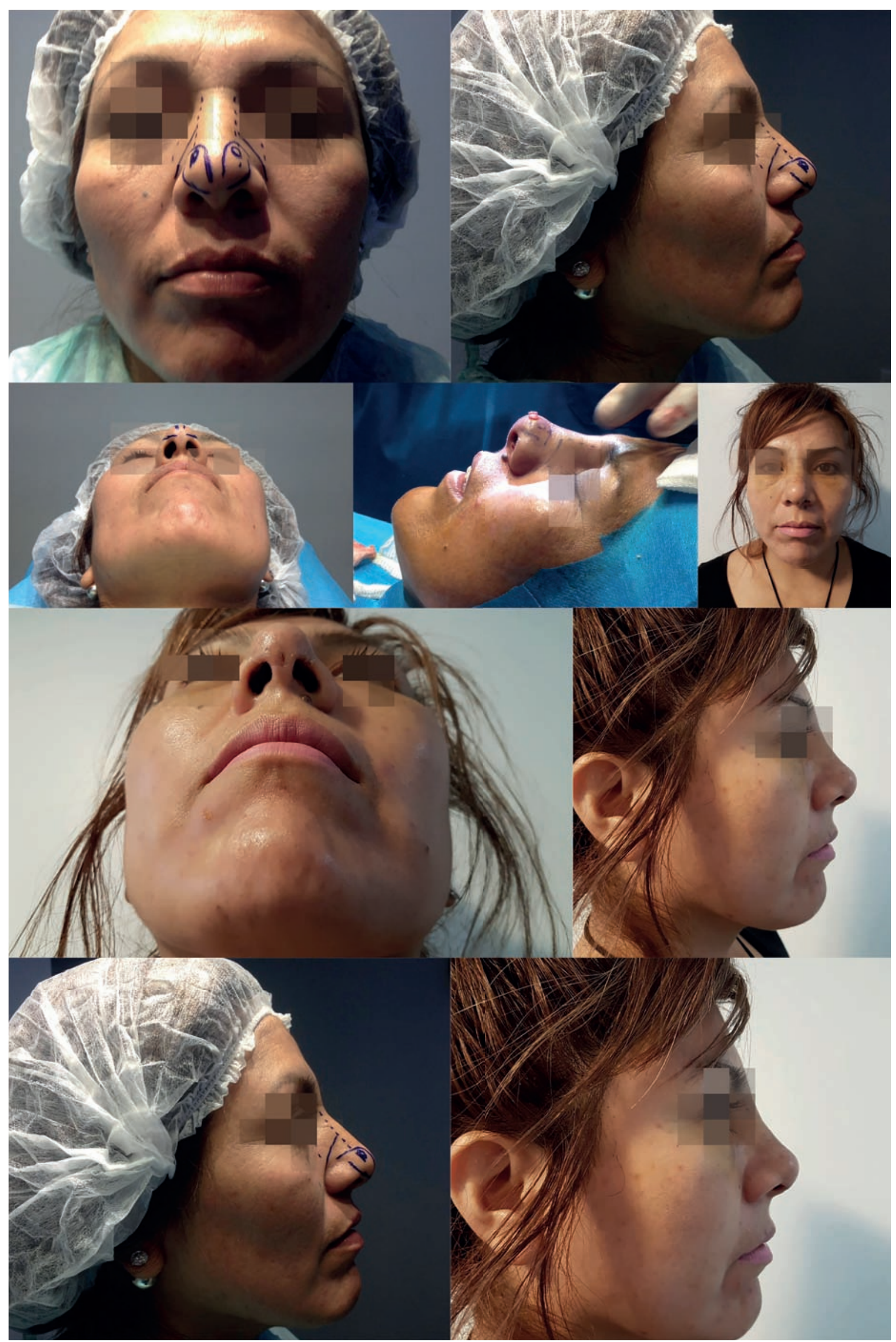

Figura 8. . Rinomodelación con punch de los domos alares 
caso, sin comprometer el borde cefálico del alar, es decir se trata de una resección cilíndrica intracartilaginosa de los alares, de esa manera se preserva el ligamento suspensor de la punta nasal.

Los cartílagos extraídos se colocan en solución fisiológica y se realiza un punto transfixiante para cerrar el área cruenta en cada lado tomando solo tejido cartilaginoso con PDS 4-0.

Inmediatamente se observa el refinamiento de cada crura externa, se sutura la mucosa con el mismo material.

De esta manera se obtiene un refinamiento de las cruras externas, utilizándose los cartílagos extraídos como injertos individuales de los domos, para dar una mejor definición de los puntos luminosos de los mismos.

$\mathrm{Al}$ combinarse con una rinomodelación, una vez que se coloca el hilo tutor fijado en el SMAS del rádix y anudado en la columela, se procede a colocar los injertos de cartílago a través de incisiones marginales de 6-8 $\mathrm{mm}$ previamente realizadas, primero se toman los injertos con PDS 4-0, luego se pasa el hilo por una aguja recta de Poget, en este caso se exteriorizan los hilos por la dermis de la punta nasal, también se puede cargar el hilo en una aguja curva atraumática, en este caso se exterioriza el hilo por la mucosa de los alares, en ambos casos se utiliza una sonda acanalada para evitar el pellizcamiento de los tejidos adyacentes. Se cortan los hilos al tercer o quinto día del posoperatorio (PO).

\section{REFINAMIENTO DE BASE ALAR}

En el caso que esté indicado realizar solo un estrechamiento de la base alar, o como complemento de una rinomodelación, se utiliza punch de 3, 4 o $5 \mathrm{~mm}$ dependiendo del espesor del tejido alar, para obtener un refinamiento simétrico y sin involucrar la mucosa nasal. Una vez que se extraen los cilindros dermograsos (estos pueden ser utilizados para aumento del rádix, previamente desepidermizados) se realiza una sutura transfixiante de la base alar con PDS 3-4-0; previamente se realizan dos incisiones puntiformes con hoja $\mathrm{N}^{\circ} 11$ en el margen alar, luego con aguja recta cargada con PDS se realiza el punto transfixiante cerrando los espacios originados por el punch. Si es necesario, se realizan puntos adicionales individuales en cada borde alar, sin tensión, con mononilon 5-0.

\section{CONCLUSIÓN REFINAMIENTO DE LA PUNTA NASAL CON PUNCH}

- Refinamiento simétrico de las cruras externas.

- Se preserva el ligamento suspensor de la punta nasal.

- Aprovechamiento de los cartílagos como injertos individuales para cada domo alar.

\section{CONCLUSIÓN REFINAMIENTO DE LA BASE ALAR CON PUNCH}

- Estrechamiento simétrico de la base alar.

- Se previenen dehiscencia en el área mucosa del piso nasal.

- Cicatriz mínima.

\section{CASOS}

BASE ALAR ANCHA

(Figuras 5, 6, 7)

\section{RINOMODELACIÓN CON PUNCH DE LOS DOMOS ALARES}

(Figuras 8)

Fotografías y videos: Dr. José Durán, todos los derechos reservados 